\title{
A SUFFICIENT CONDITION FOR THE POSITIVE RECURRENCE OF A SEMIMARTINGALE REFLECTING BROWNIAN MOTION IN AN ORTHANT ${ }^{1}$
}

\author{
By Hong Chen \\ University of British Columbia
}

\begin{abstract}
Dupuis and Williams proved that a sufficient condition for the positive recurrence and the existence of a unique stationary distribution for a semimartingale reflecting Brownian motion in an orthant (SRBM) is that all solutions of an associated deterministic Skorohod problem are attracted to the origin. In this paper, we derive a sufficient condition under which we can construct an explicit linear Lyapunov function for the Skorohod problem. Thus, this implies a sufficient condition for the stability of the deterministic Skorohod problem. The existence of such a linear Lyapunov function is equivalent to the feasibility of a set of linear inequalities. In the two-dimensional case, we recover the necessary and sufficient conditions for the positive recurrence. Some explicit sufficient conditions are derived for the higher-dimensional case.
\end{abstract}

1. Introduction. A semimartingale reflecting Brownian motion (SRBM) evolves in a $J$-dimensional nonnegative orthant $\mathfrak{R}_{+}^{J}=\left\{x \in \Re^{J}: x \geq 0\right\}$. (The vector inequalities are interpreted componentwise throughout the paper.) The key parameters that describe an SRBM are a $J$-dimensional drift for the Brownian motion $\theta$, a $J \times J$ reflection matrix $R$ and a $J \times J$ covariance matrix for the Brownian motion $\Delta$. (A more precise definition of an SRBM is given in the next section.)

Reiman and Williams $(1988,1989)$ first studied SRBMs, and they gave a necessary condition for the existence of an SRBM. This necessary condition is proved to be sufficient by Taylor and Williams (1993). This necessary and sufficient condition is that the matrix $R$ is completely- $S$ (this notion is defined in the next section). It was shown by Dupuis and Williams (1994) that an SRBM is positive recurrent and has a unique stationary distribution if all solutions of an associated deterministic Skorohod problem (SP) are attracted to the origin. Readers are referred to Dupuis and Williams (1994) for the motivation for studying SRBMs.

In this paper, a sufficient condition is obtained for the existence of a linear Lyapunov function for the deterministic Skorohod problem of Dupuis and Williams (1994). Thus, this implies a sufficient condition for positive recurrence of an SRBM. In particular, this condition recovers the necessary and

Received June 1995; revised December 1995.

${ }^{1}$ Supported in part by a grant from NSERC (Canada).

AMS 1991 subject classifications. Primary 60J60; secondary 60J65, 60K25, 34D20.

Key words and phrases. Semimartingale reflecting Brownian motion on an orthant, positive recurrence, stationary distribution, linear Lyapunov function, fluid network. 
sufficient conditions for the case $J=2$, previously derived by Hobson and Rogers (1993) and Williams (1985), and for the case when $R$ is an $M$-matrix, previously derived by Harrison and Williams (1987).

Lyapunov functions have also been used to study the stability of fluid models of queueing networks. For example, Chen (1995) used a quadratic Lyapunov function, and Dai and Weiss (1996) and Down and Meyn (1994) used a piecewise linear Lyapunov function.

In the next section, the main results are presented and they are proved in Section 3 . We conclude this introduction with some notation and conventions used throughout the paper. All vectors in $\Re^{J}$ are assumed to be column vectors. Let $\mathscr{J}=\{1, \ldots, J\}$ and suppose that $a, b \subseteq \mathscr{J}$. Then $|a|$ denotes the cardinality of $a$ and for $u \in \Re^{J}, u_{a}$ is an $|a|$-dimensional vector whose elements are those in $u$ with indices in $a$. For a $J \times J$ matrix $R, R_{a b}$ is an $|a| \times|b|$ matrix whose elements are from $R$ with row indices in $a$ and column indices in $b$, and $R_{a}$ is short for $R_{a a}$.

2. Main results. Let $R$ be a $J \times J$ matrix and $\mathscr{C}^{J}$ be the set of continuous functions from $[0, \infty)$ to $\Re^{J}$.

Definition 2.1. Given $X \in \mathscr{C}^{J}$ with $X(0) \geq 0$, the pair $Y \in \mathscr{C}^{J}$ and $Z \in \mathscr{C}^{J}$ is said to solve the SP for $X$, or simply, said to solve $\operatorname{SP}(X)$, if they jointly satisfy

$$
\begin{gathered}
Z(t)=X(t)+R Y(t) \geq 0 \quad \text { for all } t \geq 0, \\
Y(\cdot) \text { is nondecreasing with } Y(0)=0, \\
\int_{0}^{\infty} Z_{j}(t) d Y_{j}(t)=0, \quad j=1, \ldots, J .
\end{gathered}
$$

The equation (3) means that $Y_{j}(\cdot)$ can have a point of increase at $t$ only when $Z_{j}(t)=0$. A Skorohod problem $\operatorname{SP}(X)$ is said to be a linear Skorohod problem (LSP) with rate $\theta$ if $X$ takes the form $X(t)=X(0)+\theta t$; this Skorohod problem is denoted by $\operatorname{LSP}(\theta)$ with initial state $X(0)$.

A function $X \in \mathscr{b}^{J}$ is said to be attracted to the origin if for every $\varepsilon>0$, there exists a $T<\infty$ such that $|X(t)|<\varepsilon$ for all $t \geq T$.

DEFINITION 2.2. A linear Skorohod problem $\operatorname{LSP}(\theta)$ with initial state $X(0)$ is said to be stable if the $Z$ component of all of its solutions is attracted to the origin. If $\operatorname{LSP}(\theta)$ is stable for every initial state $X(0) \geq 0$, then we simply say that $\operatorname{LSP}(\theta)$ is stable.

DEFINITION 2.3. Let $\theta$ be a $J$-dimensional vector and $\Delta$ be a $J \times J$ nondegenerate covariance matrix. A semimartingale reflected Brownian motion (SRBM) is a continuous $\mathscr{F}_{t}$-adapted $J$-dimensional process $z=\{z(t), t \geq 0\}$, together with a family of probability measures $\left\{P_{x}, x \in \Re_{+}^{J}\right\}$, defined on 
some filtered probability space $\left(\Omega, \mathscr{F},\left\{\mathscr{F}_{t}\right\}\right)$ such that for each $x \in \Re_{+}^{J}$, un$\operatorname{der} P_{x}, z$, together with an $\mathscr{F}_{t}$-adapted process $y=\{y(t), t \geq 0\}$, solves $\mathrm{SP}(\{w(t)+\theta t, t \geq 0\})$, where $w=\{w(t), t \geq 0\}$ is a $J$-dimensional Brownian motion $\mathscr{T}_{t}$-martingale with covariance matrix $\Delta$ such that $w(0)=x P_{x}$-a.s.

DEFINITION 2.4. A square matrix $R$ is called an $S$-matrix if there exists a positive vector $u$ such that $R u>0$; it is called a completely-S matrix if all of its principal submatrices are $S$-matrices.

It was shown in Bernard and El Kharroui (1991) and Mandelbaum and Van der Heyden (1987) that when $R$ is completely- $S$, the Skorohod problem has at least one (pathwise) solution for any given continuous path $X \in C^{J}$, $X(0) \geq 0$; however, uniqueness may not hold, even when $R$ is a $P$-matrix. On the other hand, working with a notion of "weak" solution, Taylor and Williams (1993) proved that a sufficient condition for an SRBM to exist and to be unique in law is that the matrix $R$ be completely-S. [Berman and Plemmons (1979) and Cottle, Pang and Stone (1992) are good references for various notions of matrices mentioned in this paper.]

In Dupuis and Williams (1994), it is proved that an $\operatorname{SRBM}(\theta, R, \Delta)$ is positive recurrent and has a unique stationary distribution if the corresponding linear Skorohod problem $\operatorname{LSP}(\theta)$ is stable. Our main result is a sufficient condition for the stability of $\operatorname{LSP}(\theta)$ and hence the positive recurrence and the existence of a unique stationary distribution for $\operatorname{an} \operatorname{SRBM}(\theta, R, \Delta)$.

THEOREM 2.5. Assume that $R$ is completely-S. Then an $\operatorname{SRBM}(\theta, R, \Delta)$ is positive recurrent and has a unique stationary distribution if there exists a positive vector $h \in \mathfrak{R}^{J}$ such that given any partition $(a, b)$ of $\mathscr{J}$,

$$
h_{a}^{\prime}\left[\theta_{a}+R_{a b} u\right]<0
$$

for all $u \in\left\{v \in \Re_{+}^{|b|}: \theta_{b}+R_{b} v=0\right\}$.

REMARKS.

1. Inequality (4) is interpreted as $h^{\prime} \theta<0$ when $a=\mathscr{J}$ (and necessarily $b=\varnothing$ ), and the inequality (4) is assumed always to hold when $a=\varnothing$. In addition, the inequality (4) is assumed to hold by default when the set $\left\{v \in \Re_{+}^{|b|}: \theta_{b}+\right.$ $\left.R_{b} v=0\right\}$ is empty.

2. The sufficient condition given by the theorem is derived from a linear Lyapunov function of the form, $f(t)=h^{\prime} Z(t)$, where $Z$ solves $\operatorname{LSP}(\theta)$. A different sufficient condition can be obtained by considering a quadratic Lyapunov function of the form $f(t)=Z(t)^{\prime} A Z(t)$. This sufficient condition can be stated as follows. An $\operatorname{SRBM}(\theta, R, \Delta)$ is positive recurrent and has a unique stationary distribution if there exists a symmetric and strictly copositive matrix $A$ such that given any partition $(a, b)$ of $\mathscr{J}$,

$$
A_{a}\left[\theta_{a}-R_{a b} u\right]<0
$$


for all $u \in\left\{v \in \mathfrak{R}_{+}^{|b|}: \theta_{b}+R_{b} v=0\right\}$. [A $K \times K$ symmetric matrix $A$ is called a strictly copositive matrix, if for all $x \in \Re^{K}$ and $x \geq 0, x^{\prime} A x \geq 0$ and $x^{\prime} A x=0$ only when $x=0$. See Cottle, Habetler and Lemke (1970) for more details.]

Corollary 2.6. Assume that $R$ is completely-S and that all principal submatrices of $R$ are nonsingular. Then an $\operatorname{SRBM}(\theta, R, \Delta)$ is positive recurrent and has a unique stationary distribution if there exists a positive vector $h \in \Re^{J}$ such that for all partitions $(a, b)$ of $\mathscr{J}, h_{a}^{\prime}\left[\theta_{a}-R_{a b} R_{b}^{-1} \theta_{b}\right]<0$, whenever $R_{b}^{-1} \theta_{b} \leq 0$.

This corollary is more convenient to use. Note that $R$ being completely$S$ implies that all diagonal elements of $R$ are positive. Then for the twodimensional case, the sufficient condition given by Corollary 2.6 is

$$
\theta_{1}+r_{12} r_{22}^{-1} \theta_{2}^{-}<0 \text { and } \theta_{2}+r_{21} r_{11}^{-1} \theta_{1}^{-}<0,
$$

where $u^{-}=-\min \{u, 0\}$ for any $u \in \Re$. This condition has been shown to be both necessary and sufficient for positive recurrence of an SRBM [Hobson and Rogers (1994) and Williams (1985)].

DEFinition 2.7. A $J \times J$ matrix $R$ is Schur- $S$ if all of its principal submatrices are nonsingular and there exists a positive vector $u$ such that $u_{a}^{\prime}\left[R_{a}-\right.$ $\left.R_{a b} R_{b}^{-1} R_{b a}\right]>0$ for any partition $(a, b)$ of $\mathscr{J}$.

Corollary 2.8. Suppose that $R$ is both completely-S and Schur-S. Then $R^{-1} \theta<0$ is sufficient for an $\operatorname{SRBM}(\theta, R, \Delta)$ to be positive recurrent and to have a unique stationary distribution.

\section{REMARKS.}

1. A nonsingular $M$-matrix is both completely-S and Schur-S. (See Lemma 3.1 in the next section.) Hence, when $R$ is a nonsingular $M$-matrix, Corollary 2.8 recovers the sufficiency of the necessary and sufficient condition for positive recurrence proved by Harrison and Williams (1987), namely, that $R^{-1} \theta<0$, where $R$ is a nonsingular $M$-matrix.

2. It was proved in Peterson (1991) and Chen and Zhang (1995) that the diffusion approximations exist for multiclass feedforward queueing networks and multiclass re-entrant queueing networks with a first-bufferfirst-served (FBFS) priority discipline, respectively. [The corresponding Brownian models were also studied by Harrison and Williams (1992) and Dai, Yeh and Zhou (1994), respectively.] Let $\operatorname{SRBM}(\theta, R, \Delta)$ be the diffusion limit of multiclass feedforward queueing networks or multiclass re-entrant queueing networks with first-buffer-first-served discipline. Then the matrix $R$ was shown to be a lower triangular matrix whose inverse exists and is nonnegative. We will prove in Lemma 3.2 that a lower triangular matrix whose inverse exists and is nonnegative is both completely- $S$ and 
Schur-S. (Note that such a matrix may not be an $M$-matrix.) Therefore, Corollary 2.8 implies that for a Browniam model that corresponds to a multiclass feedforward queueing network or a multiclass re-entrant queueing network with a FBFS priority discipline, $R^{-1} \theta<0$ is sufficient for its positive recurrence and its existence as a unique stationary distribution. [Dai, Yeh and Zhou (1994) had proved this result independently for the re-entrant network in its latest version.]

3. In general, a Schur- $S$ matrix is a larger class of matrices than the class of nonsingular $M$-matrices and the classes of lower triangular matrices whose inverses exist and are nonnegative. In the two-dimensional case, all $P$-matrices are Schur- $S$, but whether this holds in general is an interesting open problem.

To close this section, we note that it does not seem to have been realized previously that there is a linear Lyapunov function for the Skorohod problem for the two-dimensional case and for the case studied by Harrison and Williams (1987).

\section{Proofs of main results.}

Proof of Theorem 2.5. Let $(Y, Z)$ be a solution pair to $\operatorname{LSP}(\theta)$ with $Z(0) \geq 0$. Since $X(t)=Z(0)+\theta t$ is Lipschitz continuous, it follows from Bernard and El Kharroui (1991) that both $Y$ and $Z$ are Lipschitz continuous and hence are absolutely continuous. Let $f(t)=h^{\prime} Z(t)$. Since $h>0, Z$ is attracted to the origin if $f$ is. By the calculus of fluid models [Dai and Weiss (1996)], for $f$ to be attracted to the origin, it suffices to show that there exists an $\varepsilon>0$ such that whenever $\dot{Y}(t)$ (the derivative of $Y$ at $t$ ) exists and $f(t)>0, \dot{f}(t) \leq-\varepsilon$.

First, let $-\epsilon$ be the maximum of $h_{a}^{\prime}\left[\theta_{a}+R_{a b} u\right]$ over all $u \in S_{b}:=\{v \in$ $\left.\Re_{+}^{|b|}: \theta_{b}+R_{b} v=0\right\}$ and all partitions $(a, b)$ of $\mathscr{J}$. Since $S_{b}$ is an affine subspace and the number of the partitions is finite, it follows from (4) that $\varepsilon>0$.

Let $t$ be a point where $\dot{Y}(t)$ and hence $\dot{Z}(t)$ and $\dot{f}(t)$ exist. Let $a=$ $\left\{j: Z_{j}(t)>0\right\}$ and $b=\left\{j: Z_{j}(t)=0\right\}$. Write (1) with $X(t)=Z(0)+\theta t$ in block form:

$$
\begin{aligned}
& Z_{a}(t)=Z_{a}(0)+\theta_{a} t+R_{a} Y_{a}(t)+R_{a b} Y_{b}(t), \\
& Z_{b}(t)=Z_{b}(0)+\theta_{b} t+R_{b a} Y_{a}(t)+R_{b} Y_{b}(t) .
\end{aligned}
$$

Taking the derivative in the above yields

$$
\begin{aligned}
& \dot{Z}_{a}(t)=\theta_{a}+R_{a} \dot{Y}_{a}(t)+R_{a b} \dot{Y}_{b}(t), \\
& \dot{Z}_{b}(t)=\theta_{b}+R_{b a} \dot{Y}_{a}(t)+R_{b} \dot{Y}_{b}(t) .
\end{aligned}
$$

Since $Z_{a}(t)>0$, relation (3) implies that $\dot{Y}_{a}(t)=0$. On the other hand, since $Z_{b}$ is nonnegative and $Z_{b}(t)=0$, it must be that $\dot{Z}_{b}(t-) \leq 0$ and $\dot{Z}_{b}(t+) \geq 0$ 
whenever the left derivative and the right derivative exist. Therefore, $\dot{Z}_{b}(t)=$ 0 as we assumed $\dot{Z}(t)$ exists. Then it follows from (5) and (6) that

$$
\begin{aligned}
& \dot{Z}_{a}(t)=\theta_{a}+R_{a b} \dot{Y}_{b}(t), \\
& \dot{Z}_{b}(t)=\theta_{b}+R_{b} \dot{Y}_{b}(t)=0 .
\end{aligned}
$$

[Note that no contradiction arises if there is no $\dot{Y}_{b}(t) \geq 0$ satisfying (8); in this case, $\dot{Y}_{b}(t)$ does not exist.] Hence, $\dot{f}(t)=h_{a}^{\prime}\left[\theta_{a}+R_{a b} \dot{Y}_{b}(t)\right] \leq-\epsilon$, where we note that $\dot{Y}_{b}(t)$ must be nonnegative and satisfy (8).

Proof OF COROLlary 2.6. Since $R_{b}^{-1}$ exists, the only possible element of the set $\left\{v \in \Re_{+}^{|b|}: \theta_{b}+R_{b} v=0\right\}$ is $v=-R_{b}^{-1} \theta_{b}$. It is in $\Re_{+}^{|b|}$ if and only if $R_{b}^{-1} \theta_{b} \leq 0$. When it is, substituting $u=-R_{b}^{-1} \theta_{b}$ into (4) yields the corollary.

Proof of CoRollary 2.8. Let $h>0$ be such that $h_{a}^{\prime}\left[R_{a}-R_{a b} R_{b}^{-1} R_{b a}\right]>0$ for all partitions $a$ and $b$. In block form, we can write

$$
R^{-1}=\left(\begin{array}{cc}
{\left[R_{a}-R_{a b} R_{b}^{-1} R_{b a}\right]^{-1}} & -\left[R_{a}-R_{a b} R_{b}^{-1} R_{b a}\right]^{-1} R_{a b} R_{b}^{-1} \\
-\left[R_{b}-R_{b a} R_{a}^{-1} R_{a b}\right]^{-1} R_{b a} R_{a}^{-1} & {\left[R_{b}-R_{b a} R_{a}^{-1} R_{a b}\right]^{-1}}
\end{array}\right)
$$

(To verify this is indeed the inverse of $R$, one should multiply by $R$ on the right of the above expression for $R^{-1}$.) Hence, the block $a$ of condition $R^{-1} \theta<0$ is equivalent to

$$
\left[R_{a}-R_{a b} R_{b}^{-1} R_{b a}\right]^{-1}\left[\theta_{a}-R_{a b} R_{b}^{-1} \theta_{b}\right]<0 .
$$

Let $g_{a}^{\prime}=h_{a}^{\prime}\left[R_{a}-R_{a b} R_{b}^{-1} R_{b a}\right]$ and note that $g_{a}^{\prime}>0$. Now multiplying (10) by $g_{a}^{\prime}$ yields

$$
h_{a}^{\prime}\left[\theta_{a}-R_{a b} R_{b}^{-1} \theta_{b}\right]<0 .
$$

Then this corollary follows from Corollary 2.6.

LEMMA 3.1. A nonsingular M matrix is both completely-S and Schur-S.

PROOF. It is clear that a nonsingular $M$-matrix is completely- $S$, and we show that it is also Schur- $S$. Let $R$ be a nonsingular $M$-matrix. Then there exists a $u>0$ such that $u^{\prime} R>0$ [see, e.g., Berman and Plemmons (1979)]. In block form, $u^{\prime} R>0$ is

$$
\begin{aligned}
& u_{a}^{\prime} R_{a}+u_{b}^{\prime} R_{b a}>0, \\
& u_{a}^{\prime} R_{a b}+u_{b}^{\prime} R_{b}>0 .
\end{aligned}
$$

Note that any principal submatrices of a nonsingular $M$-matrix are nonsingular $M$-matrices. Hence, $R_{b}$ is a nonsingular $M$-matrix and $R_{b}^{-1}$ exists and is nonnegative. Since $R$ is a non-singular $M$-matrix, $R_{b a} \leq 0$. Then multiplying (12) by $R_{b}^{-1} R_{b a}$ from the right, we have

$$
u_{b}^{\prime} R_{b a} \leq-u_{a}^{\prime} R_{a b} R_{b}^{-1} R_{b a} .
$$


Combining (11) and (13) yields

$$
u_{a}^{\prime}\left[R_{a}-R_{a b} R_{b}^{-1} R_{b a}\right]>0 .
$$

Therefore, $R$ is Schur-S.

LEMMA 3.2. A lower triangular matrix whose inverse exists and is nonnegative is both completely-S and Schur-S.

PRoOF. Let $R$ be a lower triangular matrix whose inverse exists and is nonnegative. Since the inverse of a lower triangular matrix is also a lower triangular matrix, it is clear that the diagonal element of $R$ must be positive. Hence, it is clear that $R$ is completely- $S$.

In order to show that $R$ is Schur- $S$, it suffices to show that there exists a $u>0$ such that

$$
u_{a}^{\prime}\left[R_{a}-R_{a b} R_{b}^{-1} R_{b a}\right]>0,
$$

for any partition $(a, b)$ of $\mathscr{J}$. Recall that $R^{-1}$ is a lower triangular nonnegative matrix; from its block form (9), it follows that $\left[R_{a}-R_{a b} R_{b}^{-1} R_{b a}\right]^{-1}$ is a lower triangular nonnegative matrix. This implies that

$$
\tilde{R}_{a}:=R_{a}-R_{a b} R_{b}^{-1} R_{b a}
$$

is a lower triangular matrix with positive diagonal elements. Then choose

$$
\begin{aligned}
& u_{1}=1, \\
& u_{j}=\max _{\{a: j \in a\}}\left(\left[\sum_{\{i: i \in a \text { and } 1 \leq i<j\}} u_{i}\left|\left(\tilde{R}_{a}\right)_{j i}\right|\right] /\left(\tilde{R}_{a}\right)_{j j}\right), \quad j=2, \ldots, J,
\end{aligned}
$$

where the maximum is taken over all partitions $(a, b)$ of $\mathcal{J}$ with $a$ including $j$. By its definition, it is clear that such $u$ satisfies (14).

Acknowledgments. The author would like to thank R. W. Cottle and R. J. Williams for very helpful comments.

\section{REFERENCES}

Berman, A. and Plemmons, R. J. (1979). Nonnegative Matrices in the Mathematical Sciences. Academic Press, New York.

Bernard, A. and El KhaRRoui, A. (1991). Régulation de processus dans le premier orthant de $\Re^{n}$. Stochastics Stochastics Rep. 34 149-167.

CHEN, H. (1995). Fluid approximations and stability of multiclass queueing networks: workconserving disciplines. Ann. Appl. Probab. 5 637-665.

Chen, H. and ZhANG, H. (1995). Diffusion approximations for re-entrant lines with a first-bufferfirst-served priority discipline. Unpublished manuscript.

Cottle, R. W., HABETLER, G. J. and Lemke, C. E. (1970). On classes of copositive matrices. Linear Algebra Appl. 3 295-310.

Cottle, R. W., Pang, J. S. and Stone, R. E. (1992). The Linear Complementarity Problem. Academic Press, New York.

DAI, G. J. and WeISs, G. (1996). Stability and instability of fluid models for certain re-entrant lines. Math. Oper. Res. To appear. 
DAI, J. G., YEH, D. H. and ZHOU, C. (1994). The QNET method for re-entrant queueing networks with priority disciplines. Oper. Res. To appear.

Down, D. and Meyn, S. (1994). Piecewise linear test functions for stability of queueing networks. In Proceedings of the 33rd Conference on Decision and Control 2069-2074.

DuPUis, P. and Williams, R. J. (1994). Lyapunov functions for semimartingale reflecting Brownian motions. Ann. Probab. 22 680-702.

HARRISON, J. M. and WilLIAMS, R. J. (1992). Brownian models of feedforward queueing networks: quasireversibility and product form solutions. Ann. Appl. Probab. 2 263-293.

Hobson, D. G. and Rogers, L. C. G. (1994). Recurrence and transience of reflecting Brownian motion in the quadrant. Math. Proc. Cambridge Philos. Soc. 113 387-399.

MANDElbaum, A. and VAN DER HEYDEN, L. (1987). Complementarity and reflection. Unpublished manuscript.

Peterson, W. P. (1991). A heavy traffic limit theorem for network of queues with multiple customer types. Math. Oper. Res. 16 90-118.

Reiman, M. I. and Williams, R. J. (1988). A boundary property of semimartingale reflecting Brownian motions. Probab. Theory Related Fields 77 87-97.

TAYLOR, L. M. and Williams, R. J. (1993). Existence and uniqueness of semimartingale reflecting Brownian motions in an orthant. Probab. Theory Related Fields 96 283-317.

WiLliams, R. J. (1985). Recurrence classification and invariant measure for reflected Brownian motion in a wedge. Ann. Probab. 13 758-778.

FACUlTy OF COMMERCE AND Business ADMINISTRATION

UNIVERSITY OF BRITISH COLUMBIA

VANCOUVER, BC V6T $1 Z 2$

CANADA

E-MAIL: chen@hong.commerce.ubc.ca 